Cahiers de la recherche sur les droits

Cahiers

Fur les Droits fondamentaux

6 | 2008

Pouvoirs exceptionnels et droits fondamentaux

\title{
L'état d'exception aux États-Unis : le USA PATRIOT Act et autres violations « en règle » de la Constitution
}

Wanda Mastor

\section{(2) OpenEdition}

Journals

Édition électronique

URL : https://journals.openedition.org/crdf/6827

DOI : $10.4000 /$ crdf.6827

ISSN : 2264-1246

Éditeur

Presses universitaires de Caen

Édition imprimée

Date de publication : 31 décembre 2008

Pagination : 61-70

ISBN : 978-2-84133-259-5

ISSN : 1634-8842

Référence électronique

Wanda Mastor, "L'état d'exception aux États-Unis : le USA PATRIOT Act et autres violations " en règle » de la Constitution ", Cahiers de la recherche sur les droits fondamentaux [En ligne], 6 | 2008, mis en ligne le 11 décembre 2020, consulté le 14 novembre 2022. URL : http://journals.openedition.org/crdf/6827 DOI : https://doi.org/10.4000/crdf.6827 


\title{
L'état d'exception aux États-Unis: le USA PATRIOT Act et autres violations " en règle " de la Constitution
}

\author{
Wanda MASTOR \\ Professeure à I'Université de Limoges \\ Membre de I'Observatoire des mutations institutionnelles et juridiques
}

I. Le USA PATRIOT Act, un dispositif inédit dans son fondement
A. Les événements déclencheurs de la guerre contre " l'Axe du Mal»
B. Le fondement juridique de la guerre contre "l'Axe du Mal»

II. Le USA PATRIOT Act, un dispositif inédit dans son contenu

A. Un contenu attentatoire aux droits fondamentaux

B. Un contenu sous surveillance judiciaire

III. Le USA PATRIOT Act, un dispositif inédit dans sa durée

A. Les prémisses à la prolongation de l'état d'exception

B. La confirmation de la permanence de l'état d'exception

Le 16 mars 1916, Pancho Villa attaque la petite ville de Columbus au Nouveau-Mexique. C'était la première fois qu'était menacée l'intégrité du territoire des États-Unis. Puis il y eut le 11 septembre 2001. La comparaison s'arrête là. Le raid du Mexicain, dont le but était avant tout de voler des armes et des munitions, a fait 17 morts, l'effondrement des tours jumelles, 2973. La réaction des autorités américaines en 1916 peut sembler disproportionnée : pour répondre à l'invasion humiliante de 485 Mexicains, 150000 Américains franchissent le Rio Grande pour mener une expédition punitive. En 2001, la réponse juridique semble, non pas disproportionnée - l'emploi de ce terme étant d'une grande indécence envers les victimes new yorkaises - mais inédite dans son ampleur. Elle pourrait être résumée dans ces propos tenus par un éditorialiste du New York Times: «Fight terror as if there were no rules »... ${ }^{1}$. Les États-Unis, dont la culture de la liberté fascinait tant Tocqueville, ne sont pas les seuls à permettre des entraves aux droits fondamentaux au nom de l'urgence notamment. Toutes les démocraties contemporaines ont des mécanismes prévoyant, encadrant et contrôlant de telles limites. Virtuellement, les restrictions aux droits fondamentaux dans des périodes de crise-a fortiori, en temps de guerre, comme l'a qualifié le président George W. Bush - sont prévues dans les normes suprêmes. Mais la réponse exceptionnelle apportée par les autorités à des évènements exceptionnels ne saurait perdurer sous peine de perdre toute légitimité : quelle que soit l'ampleur de l'urgence

1. T. Friedman, cité par N. Strossen, «Maintaining Human Rights in a Time of Terrorism », New York Law School Journal of Human Rights, ${ }^{\circ} 19$, 2003, p. 3 . 
ayant justifié l'état d'exception, il ne faut jamais oublier que «l'exception ne doit son existence qu'à la règle de droit ${ }^{2}$. Or le USA PATRIOT Act ${ }^{3}$, censé au départ n'être que provisoire, tend à devenir le principe et non plus l'exception. Il est la meilleure illustration de la nécessité de mener une réflexion sur les limites aux limites, tant son contenu est liberticide.

Tout en donnant la définition de la liberté, l'article 4 de la Déclaration des droits de l'homme et du citoyen de 1789 en indique la limite : «La liberté consiste à pouvoir faire tout ce qui ne nuit pas à autrui ». Aucun droit ne peut s'exercer sans bornes, la théorie des droits « limités » étant « jusqu'à présent la meilleure voie à suivre pour une plus grande compréhension de la structure des droits fondamentaux ${ }^{4}$. L'hypothèse de droits fondamentaux illimités n'est d'ailleurs pas souhaitable, un tel absolutisme conduisant fatalement à leur ineffectivité. Mais le caractère « fondamental » de certains droits entraîne une suspicion sur toute tentative de restriction. Quelle autorité peut fixer des bornes aux droits fondamentaux? Pour quel motif? Dans quel but? Jusqu'à quel point ? La plupart du temps, les textes normatifs manquent de rigueur dans l'énonciation des limites possibles, tant il est vrai que la question dépasse le domaine strictement juridique. C'est ainsi que l'article 29, alinéa 2 de la Déclaration universelle des droits de l'homme énonce de manière quasi épique que les droits sont soumis aux limitations découlant « des justes exigences de la morale, de l'ordre public et du bien-être général de la société».

Par le terme de limitation, nous entendons le bornage (c'est d'ailleurs le sens du mot latin «limitatio»), les restrictions volontaires et non des entraves engendrées par les phénomènes d'exclusion, ou résultant de l'ineffectivité des garanties ${ }^{5}$. Ces restrictions peuvent avoir des fondements très différents. Globalement, de manière schématique, deux catégories d'actions sont présentées comme nécessitant une telle restriction.
La première, pouvant être qualifiée d'interne, découle de la confrontation d'un droit fondamental à un autre (ou « collision»-Grundrechtskollision - selon l'expression allemande). Toute opération de conciliation, effectuée par le constituant, le législateur ou le juge, entraîne nécessairement l'affaiblissement d'un droit par rapport à un autre. La doctrine française positiviste refuse de parler ici de hiérarchisation des droits, tout au plus de « hiérarchie matérielle ${ }^{6}$ ou de « libertés de premier et de second rang en fonction de leur degré de protection ${ }^{7}$. Aux ÉtatsUnis, c'est essentiellement par l'intermédiaire d'un «intérêt gouvernemental » (governmental interest) protégé par la loi qu'un conflit entre droits fondamentaux est possible.

La seconde restriction, externe, est justifiée par ce que nous qualifierons de manière très générale d' «évènements exceptionnels ». Certaines constitutions européennes ont explicitement prévu des restrictions aux droits fondamentaux en cas de crise grave et de mise en ouvre de pouvoirs exceptionnels ${ }^{8}$. L'Espagne fut le premier État européen à constitutionnaliser la restriction de certains droits fondamentaux pour les nécessités de la lutte contre le terrorisme. L'article 55 alinéa 2 de sa Constitution, donnant compétence au législateur organique, envisage une telle «suspension » au cours « d'investigations liées aux agissements de bandes armées ou d'éléments terroristes ». La France a également introduit cette problématique à travers les pouvoirs exceptionnels de l'article 16 de la Constitution, l'état de siège prévu par l'article 36 du même texte, auxquels il faut ajouter l'état d'urgence ${ }^{9}$, la théorie des circonstances exceptionnelles ${ }^{10}$, ou encore la sauvegarde de l'ordre public ${ }^{11}$.

Cette entrée en matière a pour objectif de rappeler que la question de la limitation des droits rendue nécessaire par des évènements extérieurs exceptionnels n'est pas propre aux États-Unis, loin s'en faut. Mais le USA PATRIOT Act, acronyme signifiant «Uniting and Strengthening America by Providing Appropriate Tools Required to Intercept and Obstruct Terrorism ${ }^{12}$, adopté au lendemain des attentats

2. F. Saint-Bonnet, «Exception, nécessité, urgence», in Dictionnaire de la culture juridique, D. Alland, S. Rials (dir.), Paris, PUF (Quadrige), 2003 , p. 673.

3. USA PATRIOT Act of 2001, Public Law, $\mathrm{n}^{\circ}$ 107-56.

4. M. Borowski, «La restricción de los derechos fundamentales», Revista español de derecho constitucional, ${ }^{\circ}$ 59, 2000, p. 56 . C’est ainsi que la doctrine allemande a considéré qu'il existait des « limitations immanentes» (immanente Schranken) à tous les droits fondamentaux.

5. Voir D. Lochak, «Les bornes de la liberté», Pouvoirs, n 84, 1998, p. 15.

6. B. Genevois, intervention lors du débat suivant l'allocution du Doyen Vedel, «La place de la Déclaration de 1789 dans le bloc de constitutionnalité », in La Déclaration des droits de l'homme et du citoyen et la jurisprudence, Paris, PUF (Recherches politiques), 1989, p. 66.

7. L'expression est du Doyen Favoreu. Au cours du débat précité, l'auteur précise qu'en faisant cette distinction, il avait « soutenu simplement qu'il y avait deux types de protection, une protection forte et une protection atténuée. Je n’institue pas une hiérarchie, mais je constate simplement qu’il y a effectivement deux types de droits, du point de vue du degré de protection dont ils bénéficieront » (ibid., p. 69). Tous ces droits sont d'égale valeur, « sauf à considérer qu'il existe une "supraconstitutionnalité" qui permettrait de faire prévaloir certaines règles sur d'autres, ce que ne reconnaît pas le droit positif» (R. Drago, «La conciliation entre principes constitutionnels», Dalloz, 1991, chronique, p. 265).

8. À l'instar de l'article 55, alinéa 2 de la Constitution espagnole ou de l'article 19 de la Constitution portugaise.

9. Défini par la loi du 3 avril 1955, concernant les hypothèses de «péril imminent résultant d'atteintes graves à l'ordre public » ou «d'évènements présentant, par leur nature et leur gravité, le caractère de calamité publique ».

10. Telle qu'elle résulte des arrêts du Conseil d’État Heyriès (CE, 28 juin 1918, Rec., p. 651) et Dames Dol et Laurent (CE, 28 février 1919, Rec., p. 208).

11. Les contours de la notion d'ordre public, source d'innombrables interrogations et suscitant d'importantes controverses, ne sont pas aisés à délimiter. Surtout, la doctrine et le juge ordinaire ou constitutionnel assimilent encore parfois l'ordre public à l'intérêt général, l'intérêt public ou l'intérêt national. La notion d'ordre public, telle qu'elle est utilisée par la plupart des juges constitutionnels, possède un champ d'application plus restreint que l'intérêt général. De plus, si, dans la jurisprudence du Conseil constitutionnel français, la sauvegarde de l'ordre public est un objectif de valeur constitutionnelle, tel n'est pas le cas de l'intérêt général. Voir N. Jacquinot, Ordre public et Constitution, thèse sous la direction du Doyen Louis Favoreu, Aixen-Provence, décembre 2000 (dactyl.)

12. «Unir et renforcer l'Amérique en fournissant les outils appropriés pour déceler et contrer le terrorisme». 
du 11 septembre 2001, va particulièrement loin dans une logique de supériorité de la lutte conte le terrorisme sur le respect des droits fondamentaux. Il faut commencer par rappeler que la remise en cause des libertés en période de crise n'est pas une première aux États-Unis. Sous la présidence de John Adams, dans un contexte de guerre, l'Alien and Sedition Act de $1798^{13}$ punissait quiconque dont les écrits étaient de nature à nuire à l'exécutif. Plus d'une vingtaine de personnes furent arrêtées sous l'empire de cette loi jusqu'à ce que Thomas Jefferson l'abroge en 1801. C'est également la liberté d'expression qui fut mise à mal par le Sedition Act de 1918, amendement à la loi sur l'espionnage (The Espionage Act of 1917) qui permettait au gouvernement d'engager des poursuites contre ceux qui critiquaient la guerre. Ces lois ont donné l'occasion à la Cour suprême de justifier ces entorses au premier amendement de la Constitution en temps de guerre. Des militants furent poursuivis pour avoir distribué des tracts exhortant les jeunes recrues à ne pas partir combattre en Europe. Dans l'arrêt Schenk v. United States de $1919^{14}$, la Cour a estimé que «la question, dans chaque cas, est de savoir si les mots utilisés le sont dans un tel contexte et avec un tel sens qu'ils créent un danger manifeste et pressant (clear and present danger) de nature à engendrer des maux que le Congrès est en droit de prévenir. C'est une question d'urgence et de degré. Bien des choses qui pourraient être dites en temps de paix s'avèrent en temps de guerre compromettre à ce point les efforts de la nation qu'il devient impossible d'en tolérer l'expression pendant la durée des combats ${ }^{15}$. C'est également au nom de ce « danger manifeste et pressant » que la Cour valida en 1951 l'Alien Registration Act, qui permettait au gouvernement de poursuivre les militants ou simples sympathisants communistes: «Le renversement par la force et la violence du gouvernement constitue sans aucun doute un intérêt suffisamment important pour que le gouvernement limite la liberté d'expression ${ }^{16}$. Doivent encore être citées parmi les dispositions liberticides la décision du président Lincoln de suspendre les recours d'habeas corpus pendant la guerre civile ou l'Executive Order du président Roosevelt de 1941, mesure ouvertement fondée sur la race ayant pour objet de maintenir les Japonais dans des camps d'internement ${ }^{17}$.

L'histoire a donc montré que les droits fondamentaux ont pu, particulièrement en temps de guerre, être bafoués par le gouvernement américain, violations qui pour certaines ont même reçu l'aval de la Cour suprême. Mais le USA PATRIOT Act est inédit à plus d'un titre. Tout d'abord, en raison de l'ampleur des évènements qui ont déclenché son élaboration (I) ; ensuite, en raison de la dimension fortement attentatoire aux libertés fondamentales, entorse d'autant plus grave qu'elle n'est pas entourée de garanties procédurales suffisantes (II) ; enfin, en raison de la durée de son application, les mesures provisoires tendant à devenir quasiment du droit commun (III).

\section{Le USA PATRIOT Act, un dispositif inédit dans son fondement}

Pratiquement en temps réel, des centaines de millions de téléspectateurs découvrent, le mardi 11 septembre 2001, l'une des tragédies les plus marquantes de l'histoire de l'humanité. L'ampleur de cet évènement, sans précédent, va servir à justifier la guerre que George W. Bush entend mener contre «l'Axe du Mal» ${ }^{18}$, slogan d'un manichéisme troublant qui n'est pas sans rappeler «l'axe Rome-Berlin » désignant les pays fidèles à l'Allemagne nazie durant la Seconde Guerre mondiale. À évènement exceptionnel, réponse exceptionnelle: en quelques jours seulement, une loi fleuve est adoptée puis promulguée au nom de la guerre que les États-Unis entendent désormais mener contre la terreur.

\section{A. Les évènements déclencheurs de la guerre contre "l'Axe du Mal "}

Trois avions commerciaux s'écrasent contre des immeubles hautement symboliques: les tours jumelles du World Trade Center à Manhattan et le Pentagone à Washington, tandis qu'un quatrième s'écrase en rase campagne à Shanksville, en Pennsylvanie. L' « urgence», traditionnellement incluse dans la définition d'une situation exceptionnelle et, partant, de la justification de pouvoirs exceptionnels, s'impose avec une cruelle évidence. Immédiatement, les vols commerciaux vers les ÉtatsUnis sont annulés, tous les vols internationaux dans leur direction détournés vers le Canada. Les aéroports de Los Angeles et San Francisco sont fermés, l'aviation civile paralysée pendant trois jours. Presque une heure après avoir été attaquée, la tour sud s'effondre; quarante minutes s'écoulent avant que ne disparaisse également la seconde, entraînant progressivement la destruction de plusieurs autres bâtiments et stations de métro sur le même site. Sur place, le mouvement de panique, auquel il faut ajouter des vols et dégradations incontrôlables, est indescriptible. Une unité de guerre bactériologique de la garde nationale des États-Unis est même sur place afin de déterminer si des germes pathogènes n'ont pas infecté l'air. Le pays avait déjà été touché par des actes terroristes : les mêmes tours en février 1993, Oklahoma City en 1995, les ambassades américaines au Kenya et en Tanzanie en 1998.

\footnotetext{
13. Alien and Sedition Act of 1798,5 Cong. Ch. 74,1 Stat 596.

14. Schenk v. United States 249 US 47 (1919).

15. Cité par E. Zoller, Grands arrêts de la Cour suprême des États-Unis, Paris, PUF (Droit fondamental) 2000, p. 362.

16. Dennis v. United States 341 US 494 (1951), cité ibid., p. 556.

17. Mesure jugée constitutionnelle par la Cour suprême dans le tristement célèbre arrêt Korematsu v. United States 323 US 214 (1944).

18. Discours sur l'état de l'Union du 29 décembre 2002. L'expression fut de toute évidence créée par le rédacteur des discours de George W. Bush, David Frum.
} 
La réaction de la communauté internationale est immédiate: le lendemain des attentats, le Conseil de sécurité des Nations Unies adopte une résolution les condamnant; la plupart des chefs d'État réagissent vivement, dans des termes à forte connotation belliqueuse. Aussi les Nations Unies appellent-elles «la communauté internationale à redoubler d'efforts pour prévenir et éliminer les actes terroristes $[. ..]{ }^{19}$ et se déclarent-elles prêtes «à prendre toutes les mesures nécessaires pour répondre aux attaques terroristes du 11 septembre 2001 et pour combattre le terrorisme sous toutes ses formes $[\ldots] »^{20}$. Avant que George W. Bush ne parle de "guerre contre la terreur " (War on Terror), le chancelier allemand Gerhard Schröder évoque également une image guerrière: « ce n'est pas là une bataille entre les civilisations, c'est une bataille pour la civilisation ${ }^{21}$, rejetant en revanche l'image de deux blocs ennemis que reprendra largement le président des États-Unis.

Tous les éléments concourent à légitimer l'adoption de normes mettant en place des pouvoirs exceptionnels : il s'agit tout à la fois d'un état d'urgence, d'exception, de crise.

\section{B. Le fondement juridique de la guerre contre "l'Axe du Mal»}

Le 14 septembre, le président déclare l'état d'urgence nationale $^{22}$ (Declaration of National Emergency by Reason of Certain Terrorist Attacks) et signe un décret (Executive Order) soumettant les forces armées de réserve à un régime d'activité $^{23}$. George W. Bush réagit aussi rapidement que ne l'avait fait Franklin Roosevelt au lendemain de l'attaque «surprise» de Pearl Harbor le 7 décembre 1941. Ce dernier avait alors demandé immédiatement au Congrès l'autorisation de déclarer la guerre au Japon impérial; l'actuel président des États-Unis passa par la même habilitation constitutionnelle, mais pour déclarer cette fois la guerre à un ennemi non clairement identifié : le «mal», la «terreur», les « terroristes». La Constitution des États-Unis, contrairement à la plupart des constitutions européennes qui ont, dans un certain sens, " constitutionnalisé» la lutte contre le terrorisme, ne prévoit pas l'application d'un quelconque régime d'exception. La norme suprême ne fait pas mention de la réserve de loi en matière d'éventuelles limitations aux droits fondamentaux que nécessiterait l'adoption de mesures exceptionnelles. Le silence de la Constitution ne signifie pas interdiction : implicitement, l'article 1 , section 9 habilite le Congrès à prendre de telles mesures pour restaurer l'ordre : « [...] Le privilège de l'ordonnance de l'habeas corpus ne pourra être suspendu, sauf dans les cas de rébellion ou d'invasion, si la sécurité publique l'exige. $[. .$.$] ».$

De manière générale, c'est la Cour suprême qui se réserve la possibilité d'imposer son contrôle sur de telles limites tant à l'égard du législateur fédéral que des législateurs étatiques.

Le 18 septembre 2001, le Congrès autorise le chef de l'exécutif à faire usage de «toute force nécessaire et appropriée » ${ }^{24}$ à l'encontre de cet «empire du mal», pour reprendre le mot de Ronald Reagan servant alors à désigner l'Union soviétique. En matière de terrorisme, peuvent être invoquées, outre des procédures de droit commun, des prérogatives tenant aux pouvoirs exceptionnels (Emergency Power) des autorités fédérales qui vont servir de point de départ à l'adoption des dispositions particulières de $2001^{25}$.

Dans les jours qui vont suivre, un corpus de 342 pages va être élaboré sous la surveillance de son plus grand promoteur, John Ashcroft, alors Attorney General, assisté du professeur de droit constitutionnel Viêt Dinh ${ }^{26}$. Les 24 et 25 octobre 2001, la Chambre des représentants puis le Sénat adoptent à une écrasante majorité ${ }^{27}$ la loi présentée sous l'acronyme USA PATRIOT Act, promulguée par le président le 26. Le débat public n'a quasiment pas existé, pas plus que les discussions au sein même du Congrès, à une période où les alertes à l'anthrax occupaient les esprits. L'adoption de cet arsenal de mesures antiterroristes est d'autant plus inédite qu'il s'accompagne dans le même temps d'une guerre sur le terrain : l'Afghanistan, siège opérationnel d'Al Quaïda, puis l'Irak quelque temps plus tard sont envahis. Le USA PATRIOT Act, versant normatif d'une "mentalité de guerre ${ }^{28}$, donne aux autorités américaines les moyens de mener à bien cette lutte à l'échelle mondiale, moyens liberticides que leurs inspirateurs justifient au nom de la priorité du but poursuivi.

19. Point $\mathrm{n}^{\circ} 4$ de la résolution 1368 adoptée le 12 septembre 2001

20. Point $\mathrm{n}^{\circ} 5$ de la résolution 1368 adoptée le 12 septembre 2001.

21. Cité par J.-F. Revel, L'obsession anti-américaine, Paris, Plon, 2002, p. 224.

22. Proclamation 7463 du 14 septembre 2001.

23. Executive Order 13223 du 14 septembre 2001.

24. «Toute force appropriée et nécessaire contre les nations, organisations ou personnes qui auraient selon lui organisé, autorisé, exécuté ou soutenu les attaques terroristes du 11 septembre 2001, ou hébergé ces personnes, afin de prévenir toute action future de terrorisme international contre les ÉtatsUnis par ces nations, organisations ou personnes » (Authorization for use of Military Force, 18 septembre 2001).

25. Voir G. Scoffoni, «Les juges et la Constitution des États-Unis à l'épreuve du terrorisme international», in Constitution et finances publiques. Études en l'honneur de Loïc Philip, Paris, Économica, 2005, p. 221.

26. À qui les pamphlets les plus récents attribuent la paternité du PATRIOT Act: voir R. Harvey, H. Volat, USA PATRIOT Act, de l'exception à la règle, Paris, Éditions Lignes et Manifestes, 2006, notamment p. 42-53, «John Ashcroft, superpatriote».

27. 357 voix contre 66 à la Chambre des représentants, 98 voix contre 1 au Sénat (celle de Russel Feingold, présenté comme un héros par les mouvements contestataires).

28. «La nouvelle loi reflète l'esprit d'état de guerre qui règne aux États-Unis, ou, si l'on veut, le nouvel ordre mondial selon Bush, basé sur une certitude absolue d'être dans le vrai » (M. Likin, «Le PATRIOT Act et la mentalité de guerre aux États-Unis », Hommes et Libertés, $\mathrm{n}^{\circ}{ }^{131}$, juillet-août-septembre 2005, p. 31). 


\section{Le USA PATRIOT Act, un dispositif inédit dans son contenu}

Outre la production d'un nouveau corpus, le USA PATRIOT Act modifie une quinzaine de lois fédérales, dont celles sur l'immigration, sur les opérations bancaires ou encore la loi de surveillance d'intelligence étrangère (Foreign Intelligence Surveillance Act de 1978 - FISA). Cette dernière exigeait notamment, en matière de collecte d'informations, une présomption suffisante (probable cause) justifiant de telles atteintes portées au respect de la vie privée, en adéquation avec le contenu du quatrième amendement. La modification opérée par la loi de 2001 impose désormais le critère bien moins exigeant des «nécessités d'une enquête criminelle» et adapte le contenu du FISA aux nouvelles technologies, les dispositifs de repérage (Trap and Trace Devices) visant également les réseaux de téléphones portables ou les courriers électroniques.

Le USA PATRIOT Act doit se lire et se comprendre en liaison avec une série de textes tout aussi controversés. Ainsi, le Protect America Act du 5 août 2007, ironiquement rebaptisé le Police America Act par l'ACLU, a modifié la loi FISA en donnant de nouveaux pouvoirs à la puissante Agence nationale de sécurité (NSA). La NSA, autorisée à surveiller, sans mandat, toutes les communications en provenance ou à destination des États-Unis, va largement utiliser cette faculté. Des révélations publiées à la une du New York Times ${ }^{29}$ ont fait état de milliers d'écoutes extrajudiciaires effectuées au lendemain du 11 septembre. Suite à ces abus, la loi FISA a de nouveau été amendée tout récemment, par le FISA Amendments Act du 10 juillet 2008. La nouvelle loi, qui remplace celle promulguée en 1978, « réhabilite » l'obligation du mandat et rend obligatoire l'aval du tribunal spécial instauré par la loi FISA pour écouter un Américain à l'étranger alors que l'approbation du ministre de la Justice suffisait auparavant. Cette loi encadre donc la pratique des écoutes téléphoniques, mais n’échappe pas pour autant à la controverse : pour éviter que ne soit brandi le veto présidentiel, une immunité rétroactive envers les sociétés de télécommunications qui avaient collaboré avec les autorités en permettant des écoutes «sauvages» a dû être votée.
Le PATRIOT Act comporte neuf titres correspondant à des axes principaux, tels que l'augmentation de la sécurité intérieure (titre I), des procédures de surveillance (titre II), la quasi-suppression des obstacles à l'investigation (titre V), le renforcement des sanctions (titre VIII) ou encore l'amélioration des procédures de renseignement (titre IX). Les libertés individuelles ont été largement diminuées au profit de la répression. Dans la plupart des pays démocratiques, c'est au législateur qu'il appartient de rendre possible de telles entorses, l'objet de la « réserve de loi » étant «de s'assurer que les limitations apportées aux libertés des citoyens ont été consenties par ceux-ci ou par leurs représentants $»^{30}$. Or, d'une part, les atteintes les plus flagrantes portées aux libertés prennent également racine dans des sources non législatives comme, par exemple, la création des tribunaux militaires. D’autre part, la réserve de loi ne saurait être ici présentée comme un «garde-fou », tant le Congrès a vidé certains droits fondamentaux de leur substance. Il ne reste plus qu'à attendre de la jurisprudence le rôle de protecteur des libertés des individus qu'elle a si souvent joué.

\section{A. Un contenu attentatoire aux droits fondamentaux}

Le contenu du USA PATRIOT Act est en conflit tant avec des droits de «fond " qu'avec des droits de procédure. De manière générale, de nouveaux dispositifs de surveillance sont créés; les mesures d'interception des communications, auparavant cantonnées à des situations exceptionnelles, sont généralisées. Au total, six amendements de la Constitution sont mis à mal par le récent dispositif antiterroriste. Trois aspects de la loi, particulièrement controversés, entrent en conflit ouvert avec des droits constitutionnels bénéficiant traditionnellement d'une forte protection.

La section 215, intitulée «Accès à certaines archives commerciales en rapport avec la lutte contre l'espionnage et le terrorisme international», autorise le gouvernement à exiger et à saisir les bases de données de toutes sortes d'institutions, y compris les bibliothèques. Ce que l'on appelle également «la clause bibliothèque» permet avec

29. E. Lichtblau,J. Risen, «Bush lets U.S. Spy on Callers without Courts», New York Times, 16 décembre 2005.

30. J. Tremau, La réserve de loi, compétence législative et Constitution, Paris - Aix-en-Provence, Économica - Presses universitaires d'Aix-Marseille (Droit public positif), 1997, p. 36. La Constitution portugaise, après avoir rappelé que « les restrictions (apportées aux droits fondamentaux) devront se limiter à celles nécessaires à la sauvegarde d'autres droits ou intérêts protégés par la Constitution », affirme en son article 18 que «les lois qui restreignent les droits, les libertés et les garanties doivent avoir un caractère général et abstrait [...] et ne peuvent avoir d'effets rétroactifs ». La loi fondamentale allemande prévoit expressément des « réserves d'ingérence législative» (Gesetzesvorbehalte) dans lesquelles le législateur peut limiter l'exercice de certains droits fondamentaux. La doctrine allemande distingue deux types de réserves d'ingérence: les réserves générales (einfacher Gesetzesvorbehalt), confiant au législateur un pouvoir général de réglementation, et les réserves qualifiées (qualifizierter Gesetzesvorbehalt), lorsque la loi fondamentale assortit la réserve de certaines conditions, ou la subordonne à la poursuite de certains buts. Tous les droits fondamentaux ne sont cependant pas assortis d'une réserve d'ingérence législative. Tel est le cas du principe d'égalité, de la liberté de croyance, de conscience et de profession de foi, du droit à l'objection de conscience, de la liberté de la science, de la recherche et de l'enseignement supérieur. Cette absence de réserve d'ingérence législative explicite n'exclut pas les possibilités de limitations mais les soumet à l'appréciation du juge : voir D. Capitant, Les effets juridiques des droits fondamentaux en Allemagne, thèse sous la direction de Michel Fromont, Paris, décembre 1996 (dactyl.), p. 157. En France, l'article 34 de la Constitution, réservant au législateur le soin de fixer les garanties fondamentales accordées aux citoyens pour l'exercice des libertés publiques, peut être considéré comme l'équivalent d'une réserve générale d'ingérence législative allemande. Le Conseil constitutionnel a ainsi précisé que le législateur pouvait limiter l'exercice des droits fondamentaux pour assurer la protection de tout «objectif d’intérêt général qu’il appartient au législateur d'apprécier » (Conseil constitutionnel, décision $n^{\circ}$ 85-198 DC du 13 décembre 1985, Amendement Tour Eiffel, RJC I-242), que «s'agissant d'une liberté fondamentale [...] la loi ne peut en réglementer l'exercice qu'en vue de le rendre plus effectif ou de le concilier avec celui d'autres règles ou principes à valeur constitutionnelle » (Conseil constitutionnel, décision n ${ }^{\circ}$ 84-181 DC des 10 et 11 octobre 1984, Entreprises de presse, RJC I-199). 
une étonnante facilité l'intrusion des autorités gouvernementales dans la sphère personnelle de l'individu: les agents du FBI (Federal Bureau of Investigation) peuvent obtenir un mandat ${ }^{31}$ dans le but d'obtenir des dossiers médicaux, financiers, des communications électroniques (SMS, e-mails) et téléphoniques, des informations sur les vidéos louées et les livres empruntés aux bibliothèques. Cette section 215 est de plus assortie de la clause dite « du bâillon » (Gag Order) interdisant à quiconque de révéler l'utilisation par le FBI de ladite section. Outre la collision avec le quatrième amendement de la Constitution, protégeant le «droit des citoyens d'être garantis dans leurs personnes, domicile, papiers et effets, contre des perquisitions et saisies déraisonnables [...] $»^{32}$, la section 215 va à l'encontre d'une série de lois sur la confidentialité des fichiers de bibliothèques qui avaient été adoptées par les États suite à des abus perpétrés par le FBI. Les bibliothécaires, premiers opposants à l'USA PATRIOT Act, rappellent que cette possibilité donnée au FBI d'exiger « la production de toutes choses tangibles (à savoir livres, archives, papiers, documents et autres) dans le cadre d'une enquête visant le terrorisme international ou des activités d'espionnage clandestines » entre également en conflit avec plusieurs textes relatifs à leur profession. Aussi la Déclaration des bibliothécaires sur les droits civiques, leur code d'éthique ou encore le Règlement sur la confidentialité des registres seraient-ils, selon l'Association américaine des bibliothèques (ALA), purement et simplement bafoués par la section 215. Ledit règlement rappelle notamment que "l'intérêt du gouvernement pour l'usage des bibliothèques reflète une équation dangereuse et fausse entre ce qu'une personne lit et ce que cette personne croit et la manière dont cette personne peut se comporter ${ }^{33}$.

Aujourd'hui, les bibliothécaires ${ }^{34}$ sont, aux côtés de certains intellectuels (notamment le PEN American Center présidé par Salman Rushdie ${ }^{35}$ ) et du puissant syndicat américain des libertés civiques (American Civil Liberties Union-ACLU), l'un des symboles de la mobilisation antiPATRIOT Act. Robert Harvey et Hélène Volat (elle-même bibliothécaire...) rapportent que sur la plupart des portes d'accès aux bibliothèques figurent des affiches prévenant l'utilisateur que son fichier pourra être consulté par le FBI sans sa permission, parfois non sans humour ${ }^{36}$.

Autre disposition également fort contestée, la section 213, plus connue sous l'appellation péjorative de «sneak and peek», permet aux autorités de perquisitionner le domicile d'une personne avant même de lui avoir fait prendre connaissance du mandat. Ce blanc-seing permettant aux agents fédéraux «de pénétrer subrepticement pour jeter un coup d'œil » dans un lieu qui est le cœur géographique de la sphère privée est en contradiction avec le quatrième amendement cité plus haut qui proscrit les perquisitions déraisonnables (unreasonable searches and seizures). Selon l'ACLU ${ }^{37}$, le FBI aurait utilisé cette possibilité offerte par la section 213 une centaine de fois dès 2001, sans la limiter de surcroît aux cas de terrorisme. Les abus constatés par la puissante association ont poussé les membres démocrates de la commission judiciaire de la Chambre des représentants à réclamer l'ouverture d'une enquête.

La section 802, quant à elle, donne une définition particulièrement large de l'activité terroriste. La notion de «terrorisme intérieur»(Domestic Terrorism) est liée à toute activité mettant en danger des vies humaines sur le territoire national et «visant à intimider la population civile ou infléchir la politique du gouvernement par le biais de destruction massive, d'assassinats ou d'enlèvements $[\ldots] »$. Comme de nombreux auteurs l'ont relevé, " une telle définition peut ainsi s'appliquer à diverses actions n'ayant que peu de choses à voir avec le terrorisme au sens habituel. Une manifestation antiguerre occasionnant des bris de verre dans un bâtiment officiel pourrait par exemple entrer dans le champ d'application de ce texte $»^{38}$.

En raison des termes employés, ce dispositif censé être spécifique peut tout aussi bien être utilisé dans le cadre d'affaires de droit commun. Par le biais d'une interprétation extensive, toute protestation à caractère politique pourrait ainsi être qualifiée de "terrorisme intérieur", alors que le premier amendement interdit notamment au Congrès de voter des lois « [...] restreignant la liberté de parole $[. .]$.$» .$

Le USA PATRIOT Act fait plus qu'entraver les libertés fondamentales protégées par la Constitution; en atteignant la substance de certaines d'entre d'elles, telles que le droit à la vie privée ou la liberté d'expression, il les dénature. Il en va de même pour des droits constitutionnels dits de «procédure ». Ainsi, pour ne citer qu'eux, le droit à l'assistance d'un avocat protégé par le sixième amendement ou la célèbre clause de Due process contenue dans les cinquième et quatorzième amendements subissent des atteintes sans précédent. La section 412 accroît considérablement les pouvoirs de l'Attorney General en matière de détention des étrangers. Sur la seule base d'un « soupçon raisonnable» (Reasonable suspicion), il peut décider d'incarcérer un individu pendant une semaine puis prolonger sa détention pendant six mois.

31. Mandat délivré par un tribunal institué dans le cadre du FISA de 1978 pour, paradoxalement, limiter les excès du gouvernement fédéral...

32. «[...] et aucun mandat ne sera délivré, si ce n'est sur présomption sérieuse, corroborée par serment ou déclaration solennelle, ni sans qu’il décrive avec précision le lieu à fouiller et les personnes ou les choses à saisir».

33. Cité par R. Harvey, H. Volat, USA PATRIOT Act..., p. 31.

34. Voir notamment le site internet http://www.librariansagainstbush.org.

35. Le 4 août 2004, les écrivains Paul Auster, Laurie Anderson, Russel Banks, Don DeLillo et Ève Ensler ont lu des textes choisis à Cooper Union rassemblés autour du titre «État d'urgence».

36. «Organisations qui ne nous ont pas rendu visite cette semaine : 1. La Croix-Rouge, 2. Les Boys-scouts, 3. United Way, 4. FBI [barré], 5. Rotary Club» (R. Harvey, H. Volat, USA PATRIOT Act..., p. 185).

37. Informations disponibles sur le site internet de l'ACLU : http://www.aclu.org.

38. G. Scoffoni, «Les juges et la Constitution des États-Unis... », p. 223. 
À toutes ces atteintes flagrantes aux droits fondamentaux contenues dans le PATRIOT Act s'ajoutent des dispositions de nature réglementaire. Le 8 octobre 2001, le président George W. Bush institue par décret l'Office of Homeland Security afin de coordonner l'action des différentes agences fédérales ${ }^{39}$. Quelques jours plus tard, l'Attorney General John Ashcroft édicte un règlement permettant la surveillance des correspondances des détenus de même que leurs conversations avec leurs avocats ${ }^{40}$. C'est également sans passer par la voie législative, au nom de sa qualité de chef des armées, que le président des ÉtatsUnis a institué des tribunaux militaires ${ }^{41}$ dans le but d'y traduire les «combattants ennemis » (Enemy combatants) ${ }^{42}$. Face à cet arsenal liberticide, une forme de « résistance » va s'organiser. Certains États, à l'instar d'Hawaï et de l'Alaska, ont voté en 2003 des résolutions s'opposant à l'application du PATRIOT Act. En août 2005, l'ACLU avait recensé plus de 400 communautés locales (dont les villes de Philadelphie et de Cleveland) et sept États refusant d'appliquer le nouveau dispositif fédéral antiterrorisme ${ }^{43}$. Il faut ajouter à cette fronde institutionnelle permise par la forme de l'État de très nombreuses plaintes individuelles déposées contre les abus de toutes sortes commis au nom du corpus de 2001. Ces recours vont permettre à la jurisprudence de réaffirmer les exigences de l'État de droit, voire de déclarer inconstitutionnelles certaines dispositions du PATRIOT Act.

\section{B. Un contenu sous surveillance judiciaire}

La remise en cause des droits fondamentaux accompagnée d'une absence de contrôle juridictionnel effectif ne pouvait qu'entraîner une réaction de la Cour suprême. Celle-ci se produisit en 2004, lors de trois affaires relatives à des détenus de Guantanamo. Il n'y a pas lieu ici de s'étendre sur les décisions Hamdi v. Rumsfeld ${ }^{44}$, Rumsfeld v. Padilla ${ }^{45}$ et Rasulv. Bush ${ }^{46}$ du 28 juin 2004 qui dépassent le cadre de la présente étude et ont été déjà largement commentées ${ }^{47}$. Rappelons seulement que la Cour y a réaffirmé les principes élémentaires du Due process en définissant un standard minimum de protection des droits des détenus nationaux ou étrangers. La prison de Guantanamo se situant sur un territoire relevant de la compétence des États-Unis, les détenus jouissent d'un droit d'accès aux tribunaux en vertu de l'habeas corpus dont ils ne sauraient être privés. La position de la Cour suprême pourrait se résumer par ces mots lus par le juge O'Connor dans la décision Hamdi v. Rumsfeld: "We have long since made clear that a state of war is not a blank check for the Presi$d e n t{ }^{48}$... Sous la pression de l'exécutif, le Congrès va voter deux lois en 2005 et 2006 privant les détenus de Guantanamo de recours en habeas corpus, contournant ainsi la jurisprudence de la Cour.

Nouvel épisode de ce feuilleton judiciaire, l'arrêt Boumediene v. Bush rendu le 12 juin $2008^{49}$ réaffirme le droit pour les détenus de Guantanamo de contester leur détention devant les tribunaux civils. À une courte majorité (cinq voix contre quatre), la Cour a rappelé à travers la voix du juge Kennedy que « les lois et la Constitution sont conçues pour survivre et rester en vigueur même en des circonstances extraordinaires ». Comme à son habitude, le juge dissident Scalia, accompagné des autres juges conservateurs, Thomas, Alito et le Chief Justice Roberts, a eu des mots virulents envers ses collègues majoritaires dont il a dénoncé «l'arrogance » : «Pour la première fois dans l'histoire de notre Nation, la Cour a conféré un droit à l'habeas corpus à des ennemis étrangers détenus à l'étranger par nos forces armées dans le cours d'une guerre ».

Dans un arrêt Hamdan v. Rumsfeld du 29 juin $2006^{50}$, la Haute Cour s'est cette fois penchée sur la validité du Military Order du 13 novembre 2001, estimant que la création des tribunaux militaires n'était autorisée ni par une loi fédérale ni exigée par une nécessité militaire. Sans surprise, les juges Scalia, Thomas et Alito ne se sont pas ralliés à la majorité et ont rédigé des opinions dissidentes, le Chief Justice Roberts s'étant déporté de l'affaire. George W. Bush va s'empresser de faire adopter par le Congrès une loi donnant à la création desdits tribunaux la base légale qui leur manquait. Le Military Commissions Act du 17 octobre 2006 valide ainsi les mesures réglementaires contestées par la Cour suprême ${ }^{51}$. Application va en être faite pour la première fois le 6 août 2008, à l'occasion du procès du chauffeur d'Oussama Ben Laden devant un

39. Executive Order $\mathrm{n}^{\circ} 13228$ du 8 octobre 2001, prolongé par le Homeland Security Act du 25 novembre 2005, visant à rassembler une vingtaine d'agences fédérales sous une même égide.

40. Attorney General Order n ${ }^{\circ} 2529-2001$ du 30 octobre 2001.

41. Military Order du 13 novembre 2001.

42. Military Order pris sur la base d'un décret du président Roosevelt jugé conforme à la Constitution par la Cour suprême dans un arrêt Ex parte Quirin de 1942 (317 US 1 1942). Voir G. Scoffoni, «Les juges et la Constitution des États-Unis... », p. 222 et 231; S. Gardbaum, G. Scoffoni, Chronique «ÉtatsUnis », RFDC, $\mathrm{n}^{\circ}$ 50, 2002, p. 460-461.

43. Voir la page « Reform the Patriot Act» de son site internet précité.

44. 542 US 124 S. Ct. 2633 (2004).

45. 542 US 124 S. Ct. 2711 (2004).

46. 542 US 124 S. Ct. 2686 (2004).

47. Nous ne citerons que deux commentaires très détaillés, l'un en anglais, l'autre en français: D. Golove, «United States: The Bush administration's "war on terrorism" in the Supreme Court ", I. Con, International Journal of Constitutional Law, vol. 3, n 1, 2005, p. 128-146; G. Scoffoni, « Chronique ÉtatsUnis ", RFDC, $\mathrm{n}^{\circ} 63,2005$, p. 646-651.

48. Hamdi v. Rumsfeld, 542 US 124 S. Ct. 2633, 2650 (2004).

49. Lakhdar Boumediene et al. v. George Bush, 553 US (2008).

50. 548 US (2006).

51. Notons que le colonel Morris Davis, procureur général des tribunaux militaires d'exception chargés de juger les présumés terroristes incarcérés à Guantanamo, a donné sa démission le 4 octobre 2007 sur fond de tension avec l'autorité de tutelle militaire (information obtenue sur le site internet du Wall Street Journal, http://www.wsje.com). 
tribunal militaire. Le Yéménite Salim Ben Ahmed Hamdan, reconnu coupable de "soutien matériel au terrorisme », l'accusation de " complot» ayant été en revanche rejetée, encourt la prison à vie. Les deux candidats à la Maison Blanche ont salué le verdict, Barack Obama souhaitant, contrairement à John McCain, la fin des tribunaux militaires d'exception.

La réaction la plus vive à l'encontre des dispositions liberticides de l'USA PATRIOT Act va venir non pas de la Cour suprême mais de juges de district ${ }^{52}$, qui ne vont pas hésiter à déclarer inconstitutionnelles certaines dispositions de la loi. Dans une décision datée du 28 septembre $2004^{53}$, le juge du district sud de New York, Victor Marrero, va juger non conforme à la Constitution les dispositions obligeant les fournisseurs de services internet à divulguer au FBI les données personnelles de leurs clients après simple " courrier de sécurité nationale» (National Security Letters-NSL) délivré unilatéralement par l'Attorney General. Dans l'affaire opposant l'ACLU à John Ashcroft, le juge de district a estimé que ces lettres échappant à tout contrôle judiciaire et autorisant la collecte d'informations aussi personnelles que les adresses électroniques, le contenu des e-mails ou les sites webs visités contrevenaient tant au premier amendement qu'au principe de la séparation des pouvoirs. Victor Marrero va adopter le même raisonnement dans une affaire similaire jugée le 6 septembre $2007^{54}$.

Le 17 août 2006, dans un arrêt ACLU v. NSA, le juge Anna Diggs Taylor du district du Michigan va prononcer l'inconstitutionnalité et par conséquent l'arrêt immédiat des écoutes téléphoniques sans mandat (Warrant) dans des termes particulièrement virulents à l'encontre de George W. Bush. Concluant à la violation du principe de la séparation des pouvoirs et des premier et quatrième amendements à la Constitution, la juge va préciser non sans humour qu' « il n'y a pas de monarchie héréditaire en Amérique, tout comme il n'y a pas de pouvoirs créés en-dehors de la Constitution ${ }^{55}$.

Plus récemment, le juge de la Cour de district de l'Oregon, Ann Aiken, va également prononcer l'inconstitutionnalité d'une partie de l'USA PATRIOT Act. Un avocat de Portland, converti à l'Islam, a été emprisonné et interrogé par erreur suite aux attentats de la gare d'Atocha à Madrid. Estimant que les perquisitions à son domicile et à son bureau étaient illégales, il a demandé au juge Aiken de constater que le FISA, tel qu'amendé par le PATRIOT Act, violait le quatrième amendement à la Constitution. Dans une décision Brandon Mayfield v. United States of America rendue le 26 septembre $2007^{56}$, le juge de district a estimé que «le quatrième amendement avait servi cette nation pendant 220 années, et malgré bien d'autres dangers [...]. La
Constitution contient des principes piliers que les constituants tenaient pour essentiels. Ces principes ne devraient pas pouvoir être atteints facilement au nom des nécessités du moment ${ }^{57}$.

En conséquence, elle a jugé que la présomption suffisante (probable cause) exigée par le quatrième amendement pour justifier des perquisitions n'était pas respectée par le FISA, sa modification par la loi de 2001 n'imposant que les «nécessités d'une enquête criminelle».

\section{Le USA PATRIOT Act, un dispositif inédit dans sa durée}

Le terme même de «régime d'exception» enferme l'idée du provisoire. C'est le caractère temporaire des limitations apportées aux droits fondamentaux qui sert de moyen de «défense» principal aux promoteurs desdites limitations. Dès le départ, les défenseurs du PATRIOT Act ont répondu aux attaques en brandissant l'argument du provisoire. Les dispositions les plus attentatoires aux libertés ont eu pour but de réagir à une situation d'urgence absolument évidente. D'où une sorte de double légitimité des dispositions alors votées, tirée d'une part de la nature particulièrement grave et urgente de la situation, d'autre part du caractère provisoire des mesures mises en place pour y faire face. Le constat pour les défenseurs des droits et libertés est aujourd'hui amer: les principales dispositions du PATRIOT Act ont été prolongées dans le temps, certaines étant même devenues permanentes.

\section{A. Les prémisses à la prolongation de l'état d'exception}

En 2003, un PATRIOT Act II a bien failli voir le jour. Le projet du Domestic Security Enhancement Act franchissait encore une étape dans le processus de surveillance généralisée. Étaient notamment envisagés le fichage ADN des étrangers soupçonnés de certains délits ou l'autorisation pour la CIA d'espionner des citoyens américains en-dehors de tout contrôle judiciaire. Les présidents des commissions des affaires judiciaires de la Chambre des représentants et du Sénat ont alors fait savoir à John Ashcroft que le Congrès résisterait au projet du PATRIOT Act II. Faute de pouvoir faire voter un nouveau texte, l'administration Bush va tout mettre en œuvre pour que les dispositions existantes censées n'être que provisoires soient définitivement pérennisées.

\footnotetext{
52. Il n'est pas nécessaire de rappeler que depuis les deux dernières nominations à la Cour suprême, celle-ci est à majorité conservatrice ; en revanche, les jugements que nous allons évoquer sont le fait de juges de district nommés par des présidents démocrates : Anna Diggs Taylor fut nommée par Jimmy Carter, Victor Marrero et Ann Aiken par Bill Clinton...

53. Obtenue sur le site http://www.ord.uscourts.gov/. Les trois décisions évoquées peuvent également être consultées sur le site de l'ACLU précité.

54. Voir S. Cypel, «Un juge fédéral contre le PATRIOT Act et le FBI », Le Monde, 8 septembre 2007.

55. Décision obtenue sur le site http://www.ord.uscourts.gov/.

56. Ibid.

57. Ibid., p. 42-43.
} 
Le 21 juillet 2005, la Chambre des représentants va approuver à une large majorité la prolongation de l'application des mesures du PATRIOT Act par 257 voix contre 171. Le Sénat en fait de même le 29 du même mois. Il a alors été décidé que les dispositions de ladite loi seraient prolongées de cinq semaines. La Chambre va ensuite voter une nouvelle prolongation de cinq semaines, jusqu'au 10 mars 2006. Le 7 mars 2006, par une majorité de 280 voix contre 138 , elle va adopter la version définitive d'un texte reconduisant les mesures antiterroristes du PATRIOT Act.

\section{B. La confirmation de la permanence de l'état d'exception}

La plupart des dispositions du PATRIOT Act n'avaient été votées que pour une période de quatre ans, seize articles étant censés venir à expiration le 31 décembre 2005. Mais The PATRIOT Act improvement and Reauthorization Act signé par le président le 9 mars 2006 a permis la transformation d'un grand nombre de mesures temporaires en mesures permanentes.

Parmi elles, la section 218 déjà évoquée autorisant les perquisitions secrètes au domicile d'une personne. Sont en revanche seulement renouvelées pour quatre ans - du moins, en l'état actuel des choses... - les mesures de la section 215, permettant aux agents du FBI d'avoir accès aux données les plus personnelles, sans avoir à prouver que cette recherche porte sur des faits en connexion avec une activité terroriste. Est également renouvelée l'application de la section 206 autorisant l'utilisation de connexions dites «nomades", le FBI n'ayant plus besoin d'identifier le suspect pour obtenir l'autorisation d'installer son dispositif de surveillance. Les fameuses «lettres de sécurité nationale » (NSL), à l'origine des déclarations d'inconstitutionnalité prononcées par le juge Victor Marrero, reçoivent également l'aval du Congrès pour une période plus longue. Il est apparu au cours des débats parlementaires que le gouvernement avait utilisé en moyenne 30 ooo lettres de ce genre (sorte de citation donnant accès à des données personnelles) chaque année depuis les attentats de $2001^{58}$. Le renouvellement du PATRIOT Act inscrit ainsi les mesures autrefois prises au nom de l'urgence dans la durée, et ce qui relevait de l'exception tend à devenir le principe: «Tout individu devient un terroriste potentiel dont la surveillance s'inscrit dans le cadre d'un état d'exception ${ }^{59}$.
La question du PATRIOT Act permet avec une particulière acuité d'établir la frontière devant exister entre les limitations aux droits fondamentaux et leur dénaturation à laquelle des «limites illimitées » risqueraient de conduire... Ce qu'il est convenu d'appeler, suivant la terminologie allemande, les «limites des limites » (Schrankenschranken), s'adresse avant tout au législateur ${ }^{60}$. La question de l'USA PATRIOT Act illustre les failles d'une théorie absolutiste des droits fondamentaux, en vertu de laquelle la «substance» de tels droits ne devrait jamais être touchée ${ }^{61}$. Peut-on encore dire qu'aux États-Unis, le Right of Privacy ${ }^{62}$ a un "noyau dur » intouchable, invariable au sein duquel aucune ingérence ne saurait être justifiée, quel que soit le soit le motif invoqué ? Certainement pas. Son contenu est à " géométrie variable», le gouvernement le faisant varier en fonction de l'intérêt poursuivi par les limitations. Plusieurs amendements de la Constitution des États-Unis subissent de telles entorses depuis le PATRIOT Act que la limitation qui, dans certains cas, est même devenue permanente, s'apparente à de la dénaturation. Les évènements récents ont malheureusement montré qu'il ne fallait pas attendre du Congrès ou de la Cour suprême qu'ils jouent le rôle de garde-fous en veillant à ce que la frontière entre les deux ne soit jamais dépassée. Comme nous l'avons déjà évoqué, des mouvements de résistance s'organisent progressivement. Mais ils sont confrontés à une tâche bien plus difficile car relevant du domaine de l'irrationnel : lutter contre la peur que de tels évènements se reproduisent, largement entretenue par le gouvernement. Celui-ci s'est employé à diviser la nation américaine entre ceux qui sont patriotes et ceux qui ne le sont pas, comme en témoignent notamment les affiches exhortant les citoyens à « signaler toute activité suspecte». Ce patriotisme interne se traduit à l'extérieur par une vision manichéenne du monde divisé en deux camps : «soit vous êtes avec nous, soit vous êtes contre nous» a déclaré George W. Bush quelque temps après les attaques du 11 septembre. Or «lutter contre le terrorisme impose de réduire le niveau de la terreur, pas de l'accroître ${ }^{63}$. Lors des élections présidentielles de 2004, le peuple américain a pourtant renouvelé sa confiance à un président qui avait fait de la «guerre contre la terreur» une priorité. L'avenir dira peut-être s'ils étaient conscients que les moyens employés dans ce but ont remis en cause des principes qui faisaient autrefois de ce pays la première démocratie du monde.

58. Information obtenue sur le site du Washington Post (www.washingtonpost.com) : «Senator Question Terrorism Inquiries », 7 novembre 2005, p. A 10.

59. J.-C. Paye, «États-Unis : surveillance permanente des populations », Solidarités, 28 mars 2007, disponible sur le site http://www.solidarites.ch.

60. Ainsi, l'alinéa premier de l'article 19 de la loi fondamentale allemande impose que les lois restreignant les droits fondamentaux aient un caractère général et désignent formellement le droit limité.

61. La Constitution espagnole se réfère d'ailleurs à ce « contenu essentiel» (article 53, alinéa premier), tandis que la loi fondamentale allemande énonce qu' « en aucun cas il ne doit être porté atteinte à la substance d'un droit fondamental» (article 19, alinéa 2).

62. Droit constitutionnellement protégé depuis le célèbre arrêt Griswol v. Connecticut, 381 US 479 (1965).

63. N. Chomsky, «Terrorisme, l’arme des puissants », Le Monde diplomatique, décembre 2001, p. 11. 